Rev. Biol. Trop., 36(1): 139-150, 1988

\title{
Diversidad y abundancia \\ diaria y estacional del zooplancton marino de la Isla del Caño, Costa Rica
}

Héctor M. Guzmán* y Vilma L. Obando

Escuela de Biología, Universidad de Costa Rica, San Pedro, Costa Rica.

* Dirección actual: Smithsonian Tropical Research Institute, Apdo. 2072, Balboa, Panamá.

(Recibido el 5 de marzo de 1987)

\begin{abstract}
The plankton community at Caño Island might be a mixture between neritic and oceanic plankton, due to its proximity to the mainland some of its fauna could be associated to the sorrounding reefs.

The total zooplankton (indiv. $/ \mathrm{m}^{3}$ ) and copepods $/ \mathrm{m}^{3}$ show a relationship with local rainfall; with a peak of abundance during the dry season and a decrease toward the rainy season. Copepods are the dominant group $(41-63 \%$ ). Other groups present values below $10 \%$ in abundance (fish larvae, ostracods and mysids were the most important). The number of taxa is higher during the dry season.
\end{abstract}

Para las aguas continentales e insulares tropicales, existe muy poca información sobre los cambios en abundancia y ciclos reproductivos del zooplancton (Raymont 1983). La mayoria de estos trabajos versan sobre el zooplancton de la plataforma y de bahías continentales (Youngbluth 1976, 1980). Con respecto a la fauna insular zooplanctónica asociada a arrecifes, los estudios se refieren principalmente a las diversas relaciones entre los corales y el zooplancton (Richman 1975, Porter 1974, Gottfriet y Roman 1983). Pocos autores, con la excepción de Glynn (1973) y Ferraris (1982), se han referido a los cambios en abundancia del zooplancton que rodea a los arrecifes de coral.

En arrecifes coralinos asociados con masas insulares, se ha establecido una diferencia en la composición y abundancia de la fauna zooplanctónica entre zonas lagunares y oceánicas (Sale et al. 1978, Birkeland y Smalley 1981). Comúnmente el grupo dominante dentro de esta fauna ha sido el de los copépodos, representando hasta un $90 \%$ del total para muchas áreas, entre ellas, aguas neríticas y oceánicas (Ray- mont 1983) y arrecifes (Ferraris 1982). Las islas, en este aspecto, no son una excepción (Grice y Hart 1962, Herman y Beer 1969, Sale et al. 1978, Sander y Moore 1978). Tanto para la fauna residente del arrecife, en donde predominan las formas demersales, como para aguas neríticas y oceánicas de islas y continentes, se han encontrado patrones de migración vertical en diferentes condiciones físicas y en hábitats variados (Zaret y Suffer 1976, McWilliams et al. 1981).

Es evidente que en las regiones templadas y rías existe una marcada estacionalidad en la tbundancia de zooplancton. Sin embargo, varios estudios sugieren que en zonas tropicales las pequeñas fluctuaciones estacionales constituyen el rasgo común, y en muchos casos los cambios en la composición del zooplancton pueden estar asociados con variables hidrográficas locales más que con fluctuaciones estacionales regulares (Lewis y Fish 1969).

En el presente estudio se evalúan las fluctuaciones diarias, anuales y estacionales en la abundancia relativa y composición del zooplancton de la Isla del Caño. 


\section{CUADRO 1}

Precipitación total $(\mathrm{mm})$, y promedio de temperatura $\left(\mathrm{T}^{\circ} \mathrm{C}\right)$, salinidad $(\mathrm{S} \% \mathrm{oo})$, materia particulada en suspensión (MPS; $m g / 1$ ), y coeficiente de extinción de la luz (Secchi) para los meses de muestreo en 1984 y 1985, Isla del Caño (desviación estándar entre paréntesis)

\begin{tabular}{|c|c|c|c|c|c|}
\hline Mes-año & $\mathrm{P} \mathrm{mm}$ & $\mathrm{T}^{\circ} \mathrm{C}$ & $\mathrm{S} \%$ & MPS mg/1 & Coefic. Extinc \\
\hline Enero-84 & 65.2 & $\begin{array}{l}28 \\
(0.35)\end{array}$ & $\begin{array}{l}33 \\
(0.96)\end{array}$ & $\begin{array}{c}2.4 \\
(0.71)\end{array}$ & $\begin{array}{c}0.14 \\
(0.02)\end{array}$ \\
\hline Julio-84 & 333.4 & $\begin{array}{l}27 \\
(0.00)\end{array}$ & $\begin{array}{l}30 \\
(1.32)\end{array}$ & $\begin{array}{c}5.4 \\
(1.13)\end{array}$ & $\begin{array}{c}0.30 \\
(0.06)\end{array}$ \\
\hline Setiembre- 84 & 723.8 & $\begin{array}{l}28 \\
(0.00)\end{array}$ & $\begin{array}{l}28.5 \\
(1.14)\end{array}$ & $\begin{array}{l}3.1 \\
(0.24)\end{array}$ & $\begin{array}{c}0.31 \\
(0.04)\end{array}$ \\
\hline Noviembre- 84 & 611.8 & $\begin{array}{l}27.5 \\
(0.96)\end{array}$ & $\begin{array}{l}30 \\
(0.91)\end{array}$ & $\begin{array}{c}4.2 \\
(2.13)\end{array}$ & $\begin{array}{c}0.22 \\
(0.27)\end{array}$ \\
\hline Enero-85 & 81.4 & $\begin{array}{l}28.5 \\
(1.45)\end{array}$ & $\begin{array}{l}34 \\
(0.74)\end{array}$ & $\begin{array}{c}2.2 \\
(1.65)\end{array}$ & $\begin{array}{c}0.16 \\
(0.47)\end{array}$ \\
\hline Julio-85 & 420.2 & $\begin{array}{l}27 \\
(0.00)\end{array}$ & $\begin{array}{l}31 \\
(1.03)\end{array}$ & $\begin{array}{c}4.9 \\
(0.47)\end{array}$ & $\begin{array}{c}0.29 \\
(0.08)\end{array}$ \\
\hline
\end{tabular}

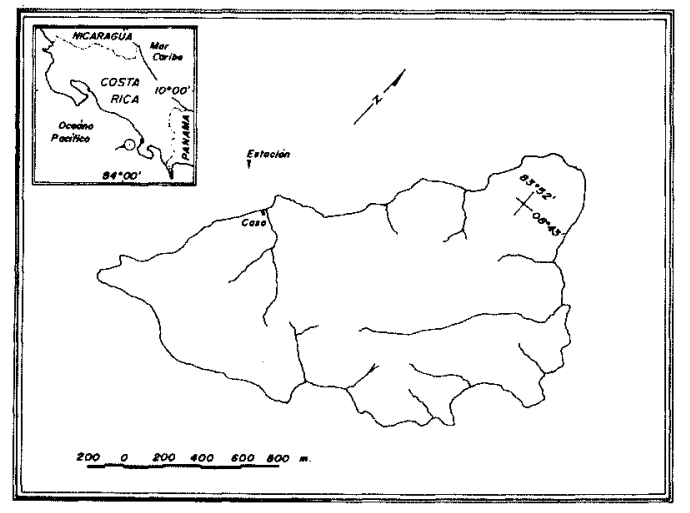

Fig. 1. Isla del Caño y estación de estudio.

Area de estudio: La Isla del Caño $\left(8043^{\prime} \mathrm{N}\right.$, $\left.85^{\circ} 52^{\prime} \mathrm{W}\right)$ se encuentra situada en el Sur del Oceáno Pacífico de Costa Rica, a una distancia de $15 \mathrm{~km}$ del Continente, al norte de la Península de Osa. Tiene una superficie de $300 \mathrm{Ha} \mathrm{y} \mathrm{el}$ perímetro de la costa es de aproximadamente $7 \mathrm{~km}$.

La altitud máxima es de $100 \mathrm{~m}$ y está cubierta por una vegetación poco alterada, típica de un bosque tropical lluvioso.
La isla se encuentra rodeada por arrecifes, con las mayores formaciones coralinas presentes en los lados Este y Norte (Guzmán 1986). Para una información más detallada de la hidrografía regional ver Dana (1975) y Guzmán (1986).

\section{MATERIAL Y METODOS}

Se estableció una estación fija al norte de la Isla, frente a la casa de Guardaparques, a 1/2 $\mathrm{km}$ aproximadamente de la costa (Fig. 1). En enero, julio, setiembre y noviembre de 1984 se tomaron muestras superficiales (un día por mes) a intervalos de 6 horas (06:00, 12:00, 18:00, 24:00 hs). En enero y julio de 1985, se tomaron muestras a intervalos de 3 horas (03:00, 06:00, 09:00, 12:00, 15:00, 18:00, 21:00, 24:00 hs).

Las muestras se tomaron por períodos de 10 minutos, a una velocidad aproximada de $2 \mathrm{nu}$ dos $(4 \mathrm{~km} / \mathrm{h})$, utilizando una red de plancton de 280 um con un diámetro de boca de $0.5 \mathrm{~m}$. El volumen filtrado se obtuvo por medio de las fórmulas $\mathrm{S}=\mathrm{V} / \mathrm{t}$, donde $\mathrm{S}$ es la distancia recorrida, $\mathrm{V}$ es la velocidad y $\mathrm{t}$ el tiempo; $\mathrm{y}$ $\mathrm{Vol}=\mathrm{AS}$, donde $\mathrm{A}$ es el área de la boca de la red. Se obtuvo un volumen aproximado de $123 \mathrm{~m}^{3}$. 


\section{CUADRO 2}

Abundancia relativa (\% de individuos) de los principales grupos $(>0.5 \%)$ a cada hora de muestreo para los meses de 1984. Isla del Caño, Osa.

\begin{tabular}{|c|c|c|c|c|c|c|c|c|c|c|c|}
\hline \multirow[b]{2}{*}{ Categoría } & \multirow[b]{2}{*}{ 06:00 } & \multicolumn{3}{|c|}{ Enero-84 } & \multicolumn{6}{|c|}{ Julio-84 } & \\
\hline & & $12: 00$ & 18:00 & $24: 00$ & Tot. (\%) & $06: 00$ & $12: 00$ & 18:00 & $24: 00$ & Tot. (\%) & \\
\hline Copepoda & 89.7 & 91.6 & 52.4 & 76.3 & 78.7 & 82.1 & 87.7 & 44.6 & 38.98 & 70.6 & \\
\hline Huevos de pez & $z \quad 1.9$ & 4.1 & 27.2 & 2.3 & 8.3 & 2.9 & 1.8 & 3.6 & - & 2.2 & \\
\hline Larvacea & 3 & - & 10 & 3.8 & 4.1 & 0.64 & - & - & - & - & \\
\hline Zoeas & 2.8 & 0.5 & 0.9 & 6.6 & 2.6 & 1.8 & 1.6 & 5.1 & 3.6 & 2.6 & \\
\hline Chaetognatha & 1.02 & 0.5 & 3.6 & 3.24 & 1.9 & 5.8 & 2 & 0.52 & - & 2.3 & \\
\hline Polychaeta & - & - & 3.9 & 0.7 & 1.2 & - & - & - & - & - & \\
\hline Larvas de pez & - & 1.03 & - & 0.8 & 0.6 & - & - & - & - & - & \\
\hline Isopoda & - & - & - & 2.8 & 0.7 & - & - & 1.3 & 3.9 & 0.9 & \\
\hline Thaliacea & - & - & - & - & 0.6 & - & - & - & - & - & \\
\hline Pelecypoda & - & - & - & - & - & 1.7 & 3.2 & 1.1 & 0.9 & 2.1 & \\
\hline Gastropoda & - & - & - & - & - & 1.1 & 1.2 & 0.9 & - & 1.01 & \\
\hline Medusas & - & 0.73 & 0.55 & - & - & 0.9 & - & - & - & - & \\
\hline Mysidacea & - & - & - & - & - & - & - & 40.5 & 48.1 & 15.1 & \\
\hline Ostracoda & - & - & - & - & -- & - & - & - & - & 0.5 & \\
\hline \multirow[t]{2}{*}{$\begin{array}{l}\text { Larvas de } \\
\text { Stomatopoda }\end{array}$} & - & - & - & - & - & - & - & - & 2.3 & - & \\
\hline & & \multicolumn{3}{|c|}{ Setiembre- 84} & \multicolumn{6}{|c|}{ Noviembre-84 } & \\
\hline Categoria & 06:00 & $12: 00$ & $18: 00$ & $24: 00$ & Tot. (\%) & $06: 00$ & $12: 00$ & $18: 00$ & $24: 00$ & Tot. $(\%)$ & $T(\%) / a n ̃ o$ \\
\hline Copepoda & 19.8 & 31.9 & 55.9 & 65.5 & 41.5 & 85.6 & 79.5 & 44.2 & 36 & 63.1 & 63.5 \\
\hline Huevos de pez & 3.1 & 5.3 & 4.5 & 4.4 & 4.3 & 3.2 & 6.8 & 12.4 & 2.1 & 3.8 & 6.7 \\
\hline Larvacea & 4.6 & 4.4 & 1.5 & 4 & 3.6 & 0.9 & 0.6 & - & 6.5 & 2.9 & 0.7 \\
\hline Zoeas & 2.7 & 7.8 & 2.1 & 4 & 4.2 & 6.5 & 7.3 & 18.2 & 32 & 17 & 6.6 \\
\hline Chae tognatha & 1.8 & 1.9 & 2.1 & 4.2 & 2.4 & 0.8 & 1.7 & - & 4.9 & 2,4 & 2.2 \\
\hline Polychacta & 9.1 & 1.3 & 1.6 & - & 3.3 & - & - & - & - & - & 1.1 \\
\hline Larvas de pez & - & - & 1.2 & - & 0.5 & - & - & 0.6 & - & - & 0.3 \\
\hline Isopoda & 2.5 & - & - & - & 0.8 & - & 6.8 & - & 1.15 & 1 & 0.9 \\
\hline Thaliacea & 1.2 & - & - & - & 0.6 & - & 0.6 & - & - & - & 0.1 \\
\hline Pelecypoda & 2.0 & - & 3.8 & - & - & - & - & 0.6 & - & - & 0.5 \\
\hline Gastropoda & 4.5 & 2.9 & 4.5 & - & 3.4 & - & 2.4 & 1.2 & 0.7 & 0.7 & 1.3 \\
\hline Medusas & - & - & - & - & - & - & - & - & - & - & - \\
\hline My sidacea & 10.4 & - & - & - & 2.7 & - & 13 & - & - & 1.1 & 4.7 \\
\hline Ostracoda & 38 & 38.3 & 21.2 & 15.4 & 29.4 & 0.6 & 2.8 & - & - & 6.7 & 9.1 \\
\hline $\begin{array}{l}\text { Larvas de } \\
\text { Stomatopoda }\end{array}$ & - & 1.3 & - & - & - & 1.2 & - & - & - & 0.7 & 0.2 \\
\hline
\end{tabular}

* Grupos con menos de $0.5 \%$ en abundancia: Cephalopoda (larvas), Ctenophora, Pteropoda, Foraminifera, Halacarida, Nematoda, Larvas de Cirripedia, Cumacea, Megalopas, Echinodermata (larvas), Nemertinea. 
Las muestras se fijaron con formaldehído neutralizado al $4 \%$ y se preservaron en etanol al $70 \%$. Cada muestra se dividió en ocho partes iguales por medio de un Separador Folson (McEven et al. 1954) y se seleccionó una submuestra al azar para ser revisada.

Se contaron todos los individuos y con el fin de comparar la abundancia, se determinó la biomasa de cada muestra por medio del método volumétrico o de desplazamiento (previamente se filtró por succión en un filtro miliporo para eliminar el agua intersticial, Beers 1976; K. Gocke, comunic. person.). Para evitar fuentes de variación en la estimación de la biomasa, las medidas no se hicieron en un período menor a un mes posterior a su preservación.

Se midió la temperatura superficial del agua, la salinidad (refractómetro), profundidad de Secchi (Coeficiente de Extinción de la luz, $\mathrm{K}=1.71 / \mathrm{d}$ ), la materia particulada en suspensión (según Cortés 1981).

La información meteorológica para los años de estudio, se obtuvo de la estación más cercana, situada en Palmar Sur ( $\left.80^{\circ} 57^{\prime} \mathrm{N}, 83{ }^{\circ} 28^{\prime} \mathrm{W}\right)$ a $16 \mathrm{~m}$ sobre el nivel del mar.

Se empleó un Análisis de Variancia de dos factores (Sokal y Rohlf 1969) para hora-mes, sin tomar en cuenta las interacciones. Además, se aplicó un ANDEVA ("oneway") y la prueba de Comparación Múltiple de Duncan, para años, meses y horas (sin réplicas). Las variables que se emplearon para ambos tipos de análisis fueron: número total de individuos (zooplancton total), número total de copépodos, número total de individuos de otros grupos y la biomasa. Para los análisis, los datos se transformaron en $\log (X+1)$ (Sokal y Rohlf 1969).

\section{RESULTADOS}

En general, durante el periodo de estudio hubo variaciones marcadas en las condiciones ambientales en la Isla (Cuadro 1). Se apreciaron cambios mensuales en la salinidad de 2 a $3 \%$ con máximo (ambos años) en el mes de enero (33\% en 1984, 34 \%oo en 1985) y minimo en setiembre de 1984 (28.5 \%/oo). Esto corresponde con el período de precipitación mínima y máxima $(65.2 \mathrm{~mm}$ en enero, $1984 ; 81.4 \mathrm{~mm}$ enero, 1985 y $723.8 \mathrm{~mm}$ en setiembre, respectivamente). La materia particulada en suspensión y el coeficiente de extinción de la luz no mostraron un patrón de cambio definido.
Variación estacional (mes-año) del zooplancton: Se encontraron 27 grupos taxonómicos, variando su número con el período. La abundancia relativa total $(\%)$ de los principales componentes del zooplancton, mostró una dominancia del grupo Copepoda, con porcentajes entre 41,5 y $79 \%$ (Cuadros 2 y 3). Ningún otro grupo, excepto mísidos, ostrácodos y zoeas, superaron el $10 \%$ de abundancia relativa. Los misidos presentaron el máximo en abundancia relativa en el mes de julio, seguido por un descenso en la población hacia finales de año (Cuadro 2). Por el contrario, en los 2 meses muestreados de 1985, el valor mayor es en enero y el menor en julio (Cuadro 3). En cuanto a los ostrácodos, se observó un aumento hacia los meses de invierno, con un máximo en setiembre-84. La cantidad de zoeas se mantuvo relativamente estable en los 2 primeros meses de muestreo de ambos años y presentó un incremento hacia finales de año. Otros grupos no menos importantes, fueron los quetognatos, huevos de pez y larváceos, cuyas poblaciones se mantuvieron en un porcentaje considerablemente estable durante el período estudiado (Cuadros 2 y 3 ).

En la Fig. 2 se observa que en todos los meses de muestreo se encontraron mayores densidades de copépodos $/ \mathrm{m}^{3}$ que de otros grupos de zooplancton. Tanto para el total del zooplancton $/ \mathrm{m}^{3}$ como para los copep. $/ \mathrm{m}^{3}$ y otros grupos $/ \mathrm{m}^{3}$, las mayores densidades se dieron principalmente en los meses de enero de ambos años, con una disminución entre setiembre $y$ noviembre del año 1984.

El análisis de variancia para el total de zooplancton $/ \mathrm{m}^{3}$, mostró una alta significancia entre meses $(\mathrm{p}<0.01)$ y entre años $(\mathrm{p}<0.01)$. Al comparar los años por separado, las diferencias significativas se encontraron entre los meses de setiembre, noviembre y enero-julio de 1984, y no hubo diferencia entre enero-julio de 1985 (Comparación Múltiple de Duncan). La variación de copépodos $/ \mathrm{m}^{3}$ entre meses y entre años fue significativa $(\mathrm{p}<0.01)$, pero no lo fue para otros grupos $/ \mathrm{m}^{3}$ entre años $(\mathrm{p}<0.01)$. E1 mismo patrón de comparaciones múltiples (Duncan) encontrado para densidad total de zooplancton/ $\mathrm{m}^{3}$, se dio para copep. $/ \mathrm{m}^{3}$ entre meses.

Variación diaria (horas) del zooplancton: En la Fig. 3a se observa la densidad total de zooplancton $/ \mathrm{m}^{3}$ por hora de muestreo. Esta fue relativamente alta en los meses de enero de ambos años, aunque la mayor densidad se obtuvo en el 


\section{CUADRO 3}

Abundancia relativa (\% de individuos) de los principales grupos (>0.5\%) a cada hora de muestreo para los meses de 1985. Isla del Caño, Osa

\begin{tabular}{|c|c|c|c|c|c|c|c|c|c|c|}
\hline \multirow[b]{2}{*}{ Categoría } & \multicolumn{10}{|c|}{ Enero-85 } \\
\hline & 03:00 & $06: 00$ & 09:00 & $12: 00$ & $15: 00$ & $18: 00$ & $21: 00$ & $24: 00$ & Tot. $(\%)$ & \\
\hline Copepoda & 48.8 & 81 & 85.1 & 88 & 81.5 & 89 & 36.1 & 71.8 & 73.9 & \\
\hline Zoeas & 8 & 1.6 & 0.63 & 1.6 & 1.8 & 1.5 & 3.8 & 1.8 & 2.5 & \\
\hline Mysidacea & 32 & - & - & 1.6 & - & - & 51.4 & 0.8 & 10.5 & \\
\hline Pelecypoda & 2 & 3.3 & 0.7 & - & 0.76 & 0.55 & 1.4 & 1.5 & 1.2 & \\
\hline Chaetognatha & 1.5 & 1.4 & 0.9 & 1.2 & 1.6 & 4 & 0.9 & 1.6 & 1.6 & \\
\hline Amphipoda & - & - & - & - & - & - & - & 0.9 & 0.3 & \\
\hline Larvacea & 0.5 & 1.7 & 3.2 & 0.9 & 7.2 & 0.9 & 0.7 & 5.5 & 2.15 & \\
\hline Thaliacea & - & 1 & 0.9 & - & - & - & - & 2.3 & 0.7 & \\
\hline Huevos de pez & - & 1 & 1.6 & 0.9 & 0.8 & - & - & 1.3 & 0.9 & \\
\hline Larvas de pez & - & 4.6 & 1.8 & 2.9 & 1.2 & 0.9 & - & 0.9 & 1.7 & \\
\hline Ostracoda & - & 1.14 & 0.8 & - & 1.2 & - & - & 3.4 & 0.9 & \\
\hline Medusas & 0.6 & - & 1.2 & - & 0.9 & - & - & 1.1 & 0.6 & \\
\hline Foraminifera & - & 0.6 & 1.1 & - & 0.9 & - & - & 1.1 & 0.6 & \\
\hline Gastropoda & - & 1 & 0.8 & - & 1.1 & 0.55 & - & 2.4 & 0.7 & \\
\hline Polychaeta & 0.8 & - & - & - & - & - & 0.7 & 2.5 & 0.6 & \\
\hline Isopoda & 2.6 & - & - & - & - & - & 1.9 & - & 0.6 & \\
\hline \multirow[t]{2}{*}{ Megalops } & 0.75 & - & - & - & - & - & 0.8 & - & 0.2 & \\
\hline & \multicolumn{10}{|c|}{ Julio-85 } \\
\hline Categoría & 03:00 & $06: 00$ & $09: 00$ & $12: 00$ & $15: 00$ & $18: 00$ & $21: 00$ & $24: 00$ & Tot. $(\%)$ & T/año \\
\hline Copepoda & 85.8 & 86 & 83 & 81.4 & 82.1 & 81.3 & 85.2 & 85.2 & 84.1 & 79.01 \\
\hline Zoeas & 1.6 & 2.9 & 8.2 & 13 & 14.4 & 6.8 & 1.7 & 1.5 & 5.1 & 3.8 \\
\hline Mysidacea & 4.5 & 1 & - & - & - & - & 5.5 & 6.2 & 2.7 & 6.6 \\
\hline Pelecypoda & - & - & - & - & 0.7 & - & - & - & 0.3 & 0.8 \\
\hline Chaetognatha & 0.5 & 2.8 & 2.9 & 2.7 & - & 0.87 & 1.02 & 1.1 & 1.5 & 1.6 \\
\hline Larvacea & 1.2 & 1.4 & 1.9 & - & 1 & 3 & 1.1 & 0.6 & 1.3 & 1.72 \\
\hline Thaliacea & 1 & 0.98 & - & - & - & 1.6 & - & 1.03 & 0.8 & 0.76 \\
\hline Huevos de pez & - & 0.6 & 0.6 & - & 1 & 3 & 0.6 & - & 0.71 & 0.79 \\
\hline Larvas de pez & - & 0.6 & - & - & - & 0.9 & - & - & 0.3 & 1.01 \\
\hline Ostracoda & - & 1.3 & - & - & - & 0.9 & 1.4 & - & 0.6 & 0.72 \\
\hline Medusas & 0.8 & 0.88 & 2.3 & 1.9 & - & - & 0.5 & 0.6 & 0.9 & 0.74 \\
\hline Foraminifera & - & - & - & - & - & - & - & - & - & 0.57 \\
\hline Gastropoda & - & - & - & - & - & 0.55 & - & - & 0.21 & 0.47 \\
\hline Polychaeta & - & - & - & - & - & - & - & - & - & 0.62 \\
\hline Isopoda & 1.9 & - & - & - & - & - & 0.7 & 2 & 0.8 & 0.7 \\
\hline Megalopas & - & - & - & - & - & - & - & - & - & 0.22 \\
\hline Amphipoda & - & - & - & - & - & - & - & - & - & 0.26 \\
\hline
\end{tabular}

Grupos con menos de $0.5 \%$ en abundancia: Sipunculida, Cirripedia, Pteropoda, Cephalopoda (larvas), Halacarida, Larvas de Echinodermata, Nauplios. 


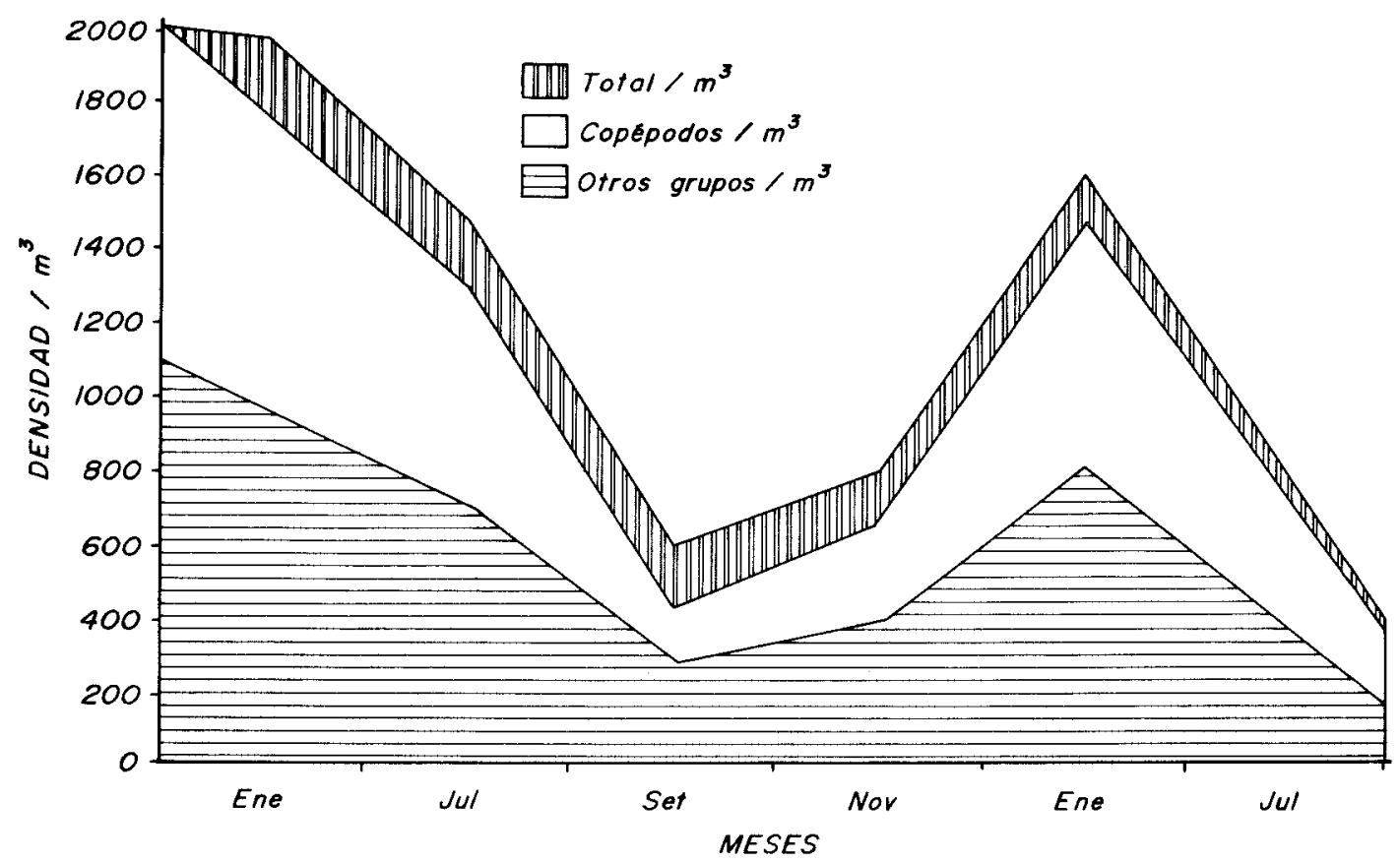

Fig. 2. Densidad (individuos $/ \mathrm{m}^{3}$ ) mensual para el total del zooplancton, copépodos y otros grupos. Isla del Caño.

mes de julio-84 a las 12 hrs. Durante 1984, se presentó una marcada reducción en setiembre, con un relativo aumento posterior (en noviembre) únicamente a las 06:00 y 24:00 hrs. El Análisis de Variancia indica una variación altamente significativa entre horas para el total de zooplancton $/ \mathrm{m}^{3}(\mathrm{p}<0,01)$.

En general no se observó un patrón definido de comportamiento diario (horas) para la densidad total de zooplancton $/ \mathrm{m}^{3}$ analizada, tanto a intervalos de 6 (1984) como de 3 horas (1985). Sin embargo, cuando dividimos el muestreo de $24 \mathrm{hrs}$ en horas diurnas (06:0015:00 hrs) y horas nocturnas (18:00-03:00 hrs), resulta que la mayor abundancia $/ \mathrm{m}^{3}$ para todos los meses de muestreo se da principalmente durante el día (Fig. 3a).

Tanto en 1984 como en 1985, la densidad aproximada de copépodos $/ \mathrm{m}^{3}$ por hora de muestreo fue generalmente mayor que la de otros organismos, excepto en setiembre-84. En enero de ambos años se obtuvieron las mayores densidades por hora de copépodos (Fig. 3b y c). Las diferencias entre horas fueron altamente significativas $(\mathrm{p}<0.01)$.

$\mathrm{Si}$ se comparan las densidades totales $/ \mathrm{m}^{3}$ (Fig. 3a) con la de copépodos $/ \mathrm{m}^{3}$ (Fig. 3b) se observa que ambas presentan un patrón similar diario de abundancia, lo cual indica que el grupo de los copépodos constituye la mayor parte del zooplancton del área. Generalmente hubo mayor densidad de copépodos que de otros organismos, tanto en horas diurnas como nocturnas.

Los Cuadros 2 y 3 muestran la abundancia relativa por hora. En enero, los copépodos dominaron a toda hora, excepto a las $21 \mathrm{hrs}$. en 1985, cuando los mísidos fueron el grupo dominante; además se observa un aumento general del porcentaje de individuos de todos los grupos en horas nocturnas (18:00-03:00 hrs). Bivalvos, larvaceos, quetognatos, zoeas, huevos y larvas de pez, aparecen todo el día, mientras que grupos como isópodos y poliquetos, se obtienen únicamente durante la noche. En julio de ambos años, los copépodos dominaron en todos los muestreos, con un aumento pronunciado en 1985. Los mísidos presentaron un incremento notable en horas de la noche, dominando a las 24:00 hrs en 1984, sin variar considerablemente en 1985. El resto de los grupos tiene una distribución diurna y nocturna similar a la de enero. Durante setiembre, el comportamiento del zooplancton cambió considerablemente; la densi- 

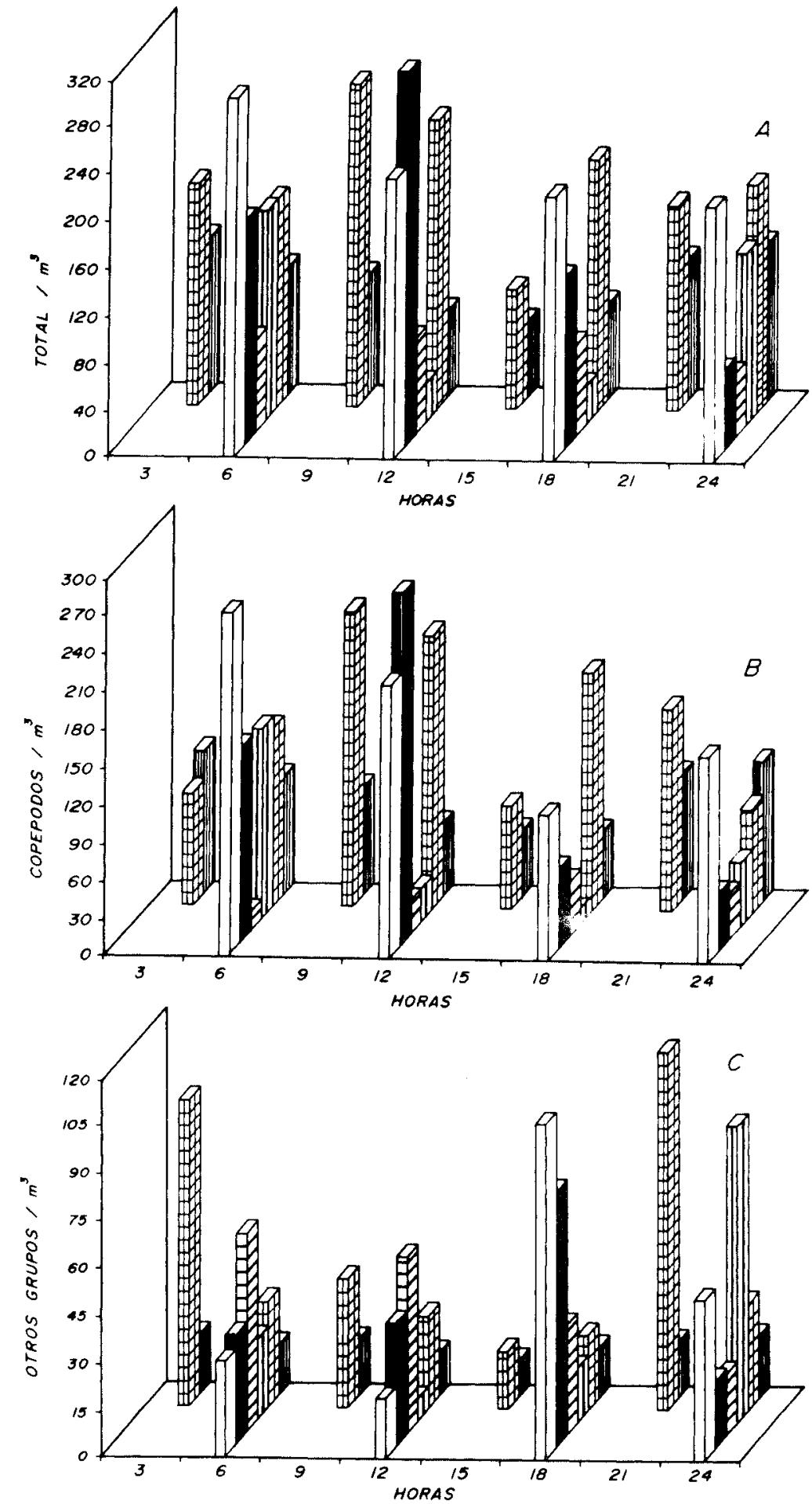

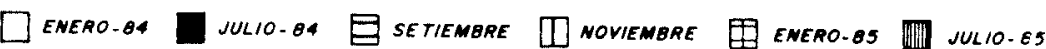

Fig. 3. Variación diaria (horas) en densidad (indiv. $/ \mathrm{m}^{3}$ ) del total de zooplancton (A), copépodos (B) y otros grupos (C) para cada mes de muestreo. Isla del Caño. 


\section{CUADRO 4}

Cantidad de grupos, densidad total aproximada (individuos $\left./ \mathrm{m}^{3}\right)$, biomasa total $\left(\mathrm{ml} / \mathrm{m}^{3}\right)$, diversidad (H), equitabilidad (J') y riqueza (SR) para todos los meses de muestreo de 1984 y 1985 , Isla del Caño (desviación estándar entre paréntesis)

\begin{tabular}{|c|c|c|c|c|c|c|}
\hline Mes-Año & Grupos & $\begin{array}{l}\text { Densidad } \\
\text { total }\end{array}$ & $\begin{array}{l}\text { Biomasa } \\
\text { total }\end{array}$ & $\mathrm{H}$ & $\mathbf{J}^{\prime}$ & SR \\
\hline Enero-84 & 23 & $\begin{array}{c}983.1 \\
(102.6)\end{array}$ & $\begin{array}{c}0.18 \\
(0.15)\end{array}$ & $\begin{array}{c}0.40 \\
(0.12)\end{array}$ & $\begin{array}{c}0.29 \\
(0.06)\end{array}$ & $\begin{array}{l}1.88 \\
(0.31)\end{array}$ \\
\hline Julio-84 & 23 & $\begin{array}{c}728.6 \\
(72.80)\end{array}$ & $\begin{array}{c}0.55 \\
(0.04)\end{array}$ & $\begin{array}{c}0.50 \\
(0.09)\end{array}$ & $\begin{array}{c}0.37 \\
(0.12)\end{array}$ & $\begin{array}{c}1.92 \\
(0.27)\end{array}$ \\
\hline Setiembre-84 & 17 & $\begin{array}{c}294.4 \\
(93.20)\end{array}$ & $\begin{array}{c}0.19 \\
(0.08)\end{array}$ & $\begin{array}{c}0.76 \\
(0.15)\end{array}$ & $\begin{array}{c}0.62 \\
(0.31)\end{array}$ & $\begin{array}{c}1.52 \\
(0.13)\end{array}$ \\
\hline Noviembre-84 & 15 & $\begin{array}{c}393.1 \\
(59.61)\end{array}$ & $\begin{array}{c}0.27 \\
(0.31)\end{array}$ & $\begin{array}{c}0.56 \\
(0.32)\end{array}$ & $\begin{array}{c}0.48 \\
(0.01)\end{array}$ & $\begin{array}{c}1.30 \\
(0.38)\end{array}$ \\
\hline Enero-85 & 21 & $\begin{array}{l}1578 \\
(226.3)\end{array}$ & $\begin{array}{c}0.38 / 1.12^{*} \\
(0.01 / 0.03)\end{array}$ & $\begin{array}{c}0.50 \\
(0.07)\end{array}$ & $\begin{array}{c}0.16 \\
(0.07)\end{array}$ & $\begin{array}{c}1.53 \\
(0.19)\end{array}$ \\
\hline Julio-85 & 23 & $\begin{array}{c}882.0 \\
(132.5)\end{array}$ & $\begin{array}{c}0.17 / 0.34^{*} \\
(0.01 / 0.06)\end{array}$ & $\begin{array}{c}0.35 \\
(0.11)\end{array}$ & $\begin{array}{c}0.11 \\
(0.02)\end{array}$ & $\begin{array}{l}1.91 \\
(1.07)\end{array}$ \\
\hline
\end{tabular}

* Incluye muestreos cada 3 horas.

dad de copépodos aumentó hacia horas de la noche. Estas variaciones coincidieron con un aumento relativo de otros organismos, como los ostrácodos, más importantes durante todo el día $(15-38 \%)$ y los mísidos, que experimentaron un aumento importante en la mañana. Poliquetos, gastrópodos y bivalvos se mantuvieron relativamente abundantes en todas las horas de muestreo y en general presentaron mayor número que en los meses anteriores. Conjuntamente, poliquetos, ostrácodos y mísidos presentaron mayores porcentajes a las 06:00 hrs, mientras que las zoeas, ostrácodos, huevos de pez y larváceos fueron más abundantes al mediodía.

El Análisis Factorial, sin tomar en cuenta las interacciones (Programa SPSS, Option 3), demostró que la gran variabilidad encontrada entre las muestras para las variables total $/ \mathrm{m}^{3}$, $\mathrm{cop} / \mathrm{m}^{3}$ y otros grupos $/ \mathrm{m}^{3}$, se da principalmente entre horas y no entre meses.

La biomasa no mostró variación significativa entre horas $(p<0.05)$, pero sí altamente significativa entre meses $(\mathrm{p}<0.01)$ y años $(\mathrm{p}<0.01)$. Comparando las medias mensuales para la biomasa (Prueba de Duncan, 0.05), se obtuvo una diferencia muy marcada para enero-85 con res. pecto al resto de los meses, que fueron similares entre sí.
Los mayores valores de diversidad y equitabilidad fueron en setiembre-84 y noviembre-84, contrario a los de Riqueza y No. de Categorías que son menores en esos meses. Para ambos años, el No. mayor de categorías y de Riqueza se dio en julio (Cuadro 4).

\section{DISCUSION}

La comunidad planctónica de la Isla posiblemente tiene una mezcla de plancton nerítico y oceánico, ya que está muy cercana a la costa. También podría pensarse que parte de esa fauna es residente debido a los arrecifes que rodean a la Isla (Aldredge y King 1977, Birkeland y Smalley 1981).

La abundancia de copépodos observada en todos los meses del estudio concuerda con observaciones realizadas a otras latitudes, con excepciones. Usualmente, este grupo constituye al menos el 70\% de la fauna planctónica (Lewis y Fish 1969; Sale et al. 1978; Youngbluth 1980; Raymont 1983). Los valores encontrados para setiembre $(41 \%)$ y noviembre (63\%), son comparables con los resultados obtenidos por Ferraris (1982) en estaciones oceánicas y dentro del arrecife de Belice. Nuestros resultados y los obtenidos en Belice, presentan porcentajes relativamente bajos si se comparan con otras áreas, 
en donde los copépodos oscilan entre el 75-80\%, como en Bermudas (Hernán y Beer 1969), Jamaica y Barbados (Lewis y Fish 1969; Moore y Sanders 1976) y Puerto Rico (Youngbluth 1980).

El resto de las categorías encontradas (por año), tuvieron una abundancia relativa menor al $10 \%$, al igual que lo obtenido en los trabajos de Ferraris (1982) y Herman y Beer (1969). Dentro de estas categorías, una de las más importantes en abundancia, fue la de los ostrácodos $(9,13 \%)$, que durante el período de "invierno" (setiembre) mantuvo un porcentaje muy alto en las dos horas de la mañana, compitiendo asi con los copépodos y otros grupos que tuvieron porcentajes mucho más bajos. Posiblemente, la reducción pronunciada de copépodos observada en setiembre fue debida a diferencias en épocas reproductivas entre estos organismos y los ostrácodos, o debido quizás a la presión de depredación ejercida por los ostrácodos, lo cual hace que este grupo sea importante en abundancia durante este mes. Por otro lado, podría pensarse que esta gran abundancia de ostrácodos está relacionada con el aumento de otro tipo de presa usada como alimento, como ciertos poliquetos $(3,2 \%$, presente estudio), crustáceos pequeños, un incremento en partículas en suspensión (Ver Cuadro 1) o algas; debido a los diversos hábitos alimenticios que presentan los ostrácodos (Barnes 1974).

El tercer grupo importante fue el ictioplancton (huevos y larvas de pez), el cual se mantuvo relativamente alto durante todo el año; Ferraris (1982) obtuvo el mismo resultado en su estudio. Generalmente, se observó una mayor abundancia en verano y durante las horas de la noche. Munro et al. (1973) encontraron una marcada estacionalidad en 83 especies de peces de arrecifes de Jamaica; durante los meses de invierno, con las temperaturas mínimas, se produjo un aumento en el número de huevos en el plancton. En los alrededores de la Isla del Caño, durante los meses de "verano", la temperatura sub-superficial disminuye drásticamente debido a la rupturas de la termoclina superficial permanente que afecta la región del Pacifico Oriental (Wyrtki 1964, 1965) durante ese período; además, los vientos y corrientes con dirección noroeste, principalmente, son fuertes en esta época (Dana 1975). Por lo tanto, podría ser posible que el aumento del ictioplancton encontrado en enero, estuviese asociado con la época reproductiva de los peces del área del arrecife, o con un transporte por medio de corrientes de otros lugares al sur del área de estudio.

Se ha encontrado en muchos trabajos, que los mísidos no son un componente importante del zooplancton (Herman y Beers 1969; Lewis y Fish 1969; Glynn 1973; Youngbluth 1980). Sin embargo, han sido observados en asociación con diferentes tipos de sustrato en los arrecifes, i.e. arenas y rocas de coral, y presentan un comportamiento migratorio nocturno muy conspicuo (Aldredge y King 1977; Porter y Porter 1977; McWilliams et al. 1981; Robinchaux et al. 1981). En el presente estudio, este grupo fue importante durante la época lluviosa. principalmente en el mes de julio y en horas de la noche, aunque en enero de 1985 abundaron en horas nocturnas no muestreadas para ese mismo mes en 1984. Robinchaux et al. (1981), describen las diferencias en comportamiento en el invierno y verano para este grupo, e indican que para el invierno, los diferentes grupos de mísidos se distribuyen uniformemente en los diversos tipos de sustrato que conforman el arrecife, mientras que en verano, encontraron que hay preferencia de ciertos grupos por determinados tipos de sustrato. Uno de nosotros (H.M.G.), ha observado que en días con aguas claras, los mísidos forman enjambres muy cercanos a las cabezas de coral, mientras que en días turbios, como el "invierno" y en cambios de marea, los enjambres se rompen y los mísidos se observan por todo el fondo.

En general, no se encontró un patrón definido en el comportamiento diario del zooplancton, ya que la abundancia de cada grupo a diferentes horas varió notablemente entre meses. Esta variabilidad parece estar relacionada con los hábitos alimenticios, los cuales causan cambios diarios en la abundancia vertical, y con los ciclos de vida que producen diferencias estacionales en la distribución vertical de cada especie (Banse 1964).

Los valores de biomasa no fueron de gran utilidad para encontrar alguna relación que explicara los cambios a través del año en la abundancia del zooplancton, ya que presentaron variaciones muy marcadas al compararlos con la densidad total (Cuadro 4). La gran variación obtenida para la biomasa en julio y noviembre de 1984 y enero de 1985 con respecto a los demás meses, se debe principalmente a la gran cantidad de mísidos, zoeas y huevos de pez que 
fueron capturados en esos períodos y no a una reducción del número de copépodos.

El número de categorías taxonómicas, fue mayor en los meses de verano con respecto al invierno. Sin embargo, la mayor diversidad y mejor distribución de los grupos se dio en el invierno. Dichos resultados y la baja abundancia en general del zooplancton en este periodo, pueden ser consecuencia de una baja captura de especies en estos meses, debido al aumento de materia orgánica en suspensión y del alga Skeletonema spp., que obstruía las redes, o bien representa el comportamiento normal de las poblaciones.

Con base en la poca información existente, se pueden hacer en general, algunas comparaciones con el área de estudio: Forsbergh y Joseph (1964) presentaron los resultados para biomasa $\left(\mathrm{ml} / \mathrm{m}^{3}\right)$ obtenidos durante el Crucero "Costa Rica Dome" (noviembre 1969) en la Isla del Coco y zonas adyacentes al Domo de Costa Rica. La biomasa para la Isla del Caño estuvo entre $0,0018-0.243 \mathrm{ml} / \mathrm{m}^{3}$ y para las regiones del Domo de Costa Rica fueron de $0.082-0.243 \mathrm{ml} / \mathrm{m}^{3}$ o valores mayores a 0.244 $\mathrm{ml} / \mathrm{m}^{3}$. Comparando dichos resultados con los valores totales mensuales del presente estudio (Cuadro 4), estos son mayores que los de la Isla del Coco y similares a los del Domo de Costa Rica. Otros cruceros como el Step-1 (set.-dic. 1960) y el Swansong (oct.-dic., 1961), realizados en zonas oceánicas al sur del Ecuador (Forsbergh y Joseph 1964) obtuvieron valores comprendidos entre 0.082 y $0.243 \mathrm{ml} / \mathrm{m}^{3}$, similares a los valores encontrados por nosotros. Vale la pena señalar que nuestro valor máximo de biomasa $\left(0.55 \mathrm{ml} / \mathrm{m}^{3}\right)$ corresponde a uno de los meses no muestreados por ninguno de los cruceros anteriormente citados, y además las diferencias encontradas posiblemente se deban a que los valores proporcionados por dichos autores corresponden a una sola muestra en un solo año.

La estacionalidad observada para el total del zooplancton y copépodos, refleja una relación clara con la precipitación local, es decir, con los cambios de estación; la mayor abundancia para el mes de enero ("verano") y la disminución en los meses de "invierno", coinciden con el comportamiento del zooplancton en general (Raymont 1983). Lewis y Fish (1969) encontraron picos de abundancia del zooplancton durante todo el año en Barbados, pero el mayor número de individuos se da en el "verano" (enero-febre- ro) y el menor en el "invierno" (julio). Nuestros resultados sugieren una posible estacionalidad con un solo pico de abundancia a lo largo del año, lo cual coincide con la mayoría de los trabajos realizados en las regiones tropicales del Caribe (Herman y Beers 1969; Lewis y Fish 1969; Glynn 1973; Youngbluth 1980). Además, coincide con la información obtenida en el Golfo de Panamá que es la única existente para la región; Forsbergh (1969) encontró que el pico de abundancia más alto del zooplancton, se da durante abril y junio, posteriores al afloramiento de esta zona. En el presente estudio ese único pico de abundancia, que podría ser común en el Pacífico Oriental Tropical, se dio durante la estación seca, ya que esta zona no está afectada por afloramientos. El pico de mayor abundancia encontrado para la primera época del año, pudo ser resultado de la combinación de dos factores, uno biológico y otro físico: el resultado eventual del surgimiento del fitoplancton ocurrido durante los meses de "invierno", produciendo un aumento gradual del zooplancton para la época de "verano". Esto posiblemente es ayudado por el fenómeno regional de la desaparición de la termoclina permanente superficial que ocurre entre los meses de diciembre a febrero principalmente, y trae quizás como consecuencia un aumento en nutrientes y por consiguiente un aumento en la productividad del área.

\section{AGRADECIMIENTOS}

Agradecemos al personal de la Reserva Biológica de la Isla del Caño su colaboración y hospitalidad durante los años de estudio y a la Estación Biológica Marenco por el apoyo logístico.

A Albert Bornesmiza por su ayuda en el análisis e interpretación de datos; a Ana Dittel y Ricardo Soto por su sustanciosa y constructiva crítica al manuscrito y a Carlos E. Jiménez por su colaboración en el muestreo. A Alberto León por la confección de las figuras y a todos los compañeros que colaboraron en el trabajo de campo.

Este proyecto fue financiado parcialmente por la Vicerrectoría de Investigación de la Universidad de Costa Rica (No. 808-83-135) y por el Dr. Peter W. Glynn (Proyecto Fundación Nacional de Ciencia, U.S.A., No. OCE-8415615). 


\section{RESUMEN}

La comunidad planctónica de la isla es posiblemente mezcla de plancton nerítico y oceánico dada su cercanía a la costa y parte de su fauna puede ser residente debido a los arrecifes que la rodean.

La estacionalidad observada para el total de zooplancton (indiv $/ \mathrm{m}^{3}$ ) y copépodos $/ \mathrm{m}^{3}$ refleja una relación con la precipitación local (cambios de estación); con una mayor abundancia en "verano" (época seca) y una disminución hacia los meses de "invierno" (época lluviosa). Los copépodos constituyen el grupo dominante, oscilando entre un 41 y un $63 \%$ durante el período de estudio. Los otros grupos encontrados tuvieron valores de abundancia total menores al $10 \%$, siendo las larvas de pez, ostrácodos, y mísidos los más importantes. El número de categorías (grupos taxonómicos) es mayor durante el verano.

\section{REFERENCIAS}

Aldredge, A.L. \& J.M. King. 1977. Distribution, abundance and substrate preference of demersal reef zooplankton at Lizard Island Lagoon, Great Barrier Reef. Mar. Biol. 41:317-333.

Banse, K. 1964. On the vertical distribution of zooplankton in the sea. Prog. Oceanogr. 2:56-125.

Barnes, R.D. 1974. Invertebrate Zoology. W.B. Saunders \& Co., Philadelphia. 870 p.

Beers, J.R. 1976. Determination of zooplankton biomasss. Monograph Oceanogr. Methodol (UNESCO). 4:35-84

Bennet, E.B. 1966. Influence of the Azores High on sea level pressure and wind, and on precipitation, in the Eastern Tropical Pacific Ocean. Inter-Amer. Trop. Tuna Comm., Bull. 12:1-23.

Birkeland, C. \& T.L. Smalley. 1981. Comparison of demersal plankton from comparable substrata from a high island and a atoll. Proc. 4th Inter. Coral Reef Symp. 1:437-442.

Cortés, J. 1981. The coral reef at Cahuita, Costa Rica: a reef under stress. M. Sc. Thesis, McMaster University, Hamilton, Ontario. 176 p.

Dana, T.F. 1975. Development of contemporary eastern pacific coral reefs. Mar. Biol. 33:355-374.

Ferraris, J.D. 1982. Surface zooplankton at Carrie Bow Cay, Belize, 1. Smith. Contrib., Mar. Sci. 12:143-151.
Forsbergh, E.D. \& J. Joseph. 1964. Biological production in the Eastern Pacific Ocean. Inter-Amer. Trop. Tuna Comm. Bull, 8:512-527.

Forsbergh, E.D. 1969. On the climatology, oceanography and fisheries of the Panama Bight. Bull. InterAmer. Trop. Tuna Comm. 14:49-385.

Glynn, P.W. 1973. Ecology of a Caribbean Coral reef, the Porites reef-flat biotope: Part II. Plankton community with evidence for depletion. Mar. Biol. $22: 1-21$

Gottfriet, M. \& M. Roman. 1983. Ingestion and incorporation of Coral-Mucus detritus by reezooplankton. Mar. Biol. 72:211-218.

Grice, G.D. \& A.D. Hart. 1962. The abundance, seasonal ocurrence and distribution of the epizooplancton between New York and Bermuda. Ecol. Monogr. 32:287-307.

Guzmán, H.M. 1986. Estructura de la comunidad arrecifal de la Isla del Caño, Costa Rica y el efecto de perturbaciones naturales severas. Tesis de Maestría, Universidad de Costa Rica. 179 p.

Herman, S. \& J.R. Beers. 1969. The ecology of inshore plankton populations in Bermuda. Part II. Seasonal abundance and composition of the zooplankton. Bull. Mar. Sci. 19:483-503.

Lewis, J.B. \& A.G. Fish. 1969. Seasonal variation of the zooplankton fauna of surface waters entering the Caribbean sea at Barbados. Car. Jour. Sci. 9:1-24.

McEven, G.F., M.W. Johnson \& T.R. Folson. 1954. A Statistical analysis of the performance of the Folson Plankton Splitter based upon test observations. Arch. Fuer Meteorologie, Geophysik und Bioklimatologie, Serie A: Meteorologie und Geophysik., Vol. 7.

McWilliam, P.S., P.E. Sale \& D.T. Anderson. 1981. Seasonal changes in resident zooplankton sampled by emergence traps iñn one tree lagoon, Great Barrier Reef. J. Exp. Mar. Biol. Ecol. 52:185-203.

Moore, E. \& F. Sander. 1976. Quantitative and qualitative aspects of zooplankton and breeding of copepods at two Caribbean coral reef stations. Est. Coastal Mar. Sci. 4:589-607.

Munro, J.L., V.C. Gaut., R. Thompson \& P.H. Reeson. 1973. The spawning seasons of Caribbean reef fishes. J. Fish Biol. 5:69-84.

Porter, J.W. \& K.G. Porter. 1977. Quantitative sampling of demersal plankton migrating from different coral reef substrates. Limm. Ocean. 22:553-555.

Porter, J.W. 1974. Zooplankton feeding by the Caribbean reef-building coral Montastrea cavernosa. Proc. Second Inter. Coral Reef Symp. 1:111-125. 
Raymont, J. 1982. Plankton and productivity in the oceans. Pergamon Press International Library. $815 \mathrm{p}$.

Richman, S., Y. Loya \& L. Slobodkin. 1975. The rate of mucus production by corals and its assimilation by the coral reef copepod Acartia negligens. Limnol-Oceanogr. 20(6).

Robichaux, D.M., A.G. Cohen., M.L. Reaka \& D. Allen. 1981. Experiments with zooplankton on coral reefs, or, will the real demersal plankton please come up?. Mar. Ecol. 2:77-94.

Sale, P.F., P.S. McWilliams \& D.I. Anderson. 1978. Faunal relationships among near-reef zooplankton at three locations on Heron Reef, Great Barrier Reef, and seasonal changes in this fauna. Mar. Biol. 49:133-145.
Sander, F. \& E. Moore. 1978. A comparative study of inshore and offshore copepod populations at Barbados, West Indies. Crustaceana 35:226-240.

Sokal, R.R. \& F.J. Rohlf. 1969. Biometry. W.H. Freeman and Co. San Francisco. 776 p.

Wyrtki, K. 1964. Upwelling in the Costa Rica Dome. J.S. Fish and wildlife service. Fish Bull. 63:355372.

Wyrtki, K. 1965. Surface currents of the Eastern Tropical Pacific Ocean. Inter-Amer. Trop-Amer. Trop. Comm Bull. 9:279-304.

Youngbluth, M.J. 1980. Daily, seasonal and annual fluctuations among zooplankton populations in an unpolluted tropical embayment. Est. Coas. Mar. Sci. 10:265-287.

Zaret, I.M. \& W.C. Suffer. 1976. Vertical migration in zooplankton as a predaton avoidance mechanism. Limm. Ocean. 21:804-814. 